\title{
The decline in adolescent substance use across Europe and North America in the early twenty-first century: A result of the digital revolution?
}

\author{
Margaretha De Looze ${ }^{1}$ S. van Dorsselaer ${ }^{2}$ G. W. J. M. Stevens ${ }^{1} \cdot$ M. Boniel-Nissim ${ }^{3} \cdot$ A. Vieno ${ }^{4} \cdot$ \\ R. J. J. M. Van den Eijnden ${ }^{1}$
}

Received: 8 May 2018/Revised: 6 November 2018/Accepted: 23 November 2018/Published online: 17 December 2018

(c) The Author(s) 2018

\begin{abstract}
Objectives Increases in electronic media communication (EMC) and decreases in face-to-face peer contact in the evening (FTF) have been thought to explain the recent decline in adolescent substance use (alcohol, tobacco, cannabis). This study addresses this hypothesis, by examining associations between (time trends in) EMC, FTF, and substance use in more than 25 mainly European countries.

Methods Using 2002-2014 data from the international Health Behaviour in School-aged Children (HBSC) study, we ran multilevel logistic regression analyses to investigate the above associations.

Results National declines in substance use were associated with declines in FTF, but not with increases in EMC. At the individual level, both EMC and FTF related positively to substance use. For alcohol and cannabis use, the positive association with EMC was stronger in more recent years. Associations between EMC and substance use varied across countries, but this variation could not be explained by the proportion of young people using EMC within countries.

Conclusions Our research suggests that the decrease in FTF, but not the increase in EMC, plays a role in the recent decrease in adolescent substance use.
\end{abstract}

Keywords Adolescence $\cdot$ Substance use $\cdot$ Tobacco $\cdot$ Alcohol $\cdot$ Cannabis $\cdot$ Electronic media communication .

Internet $\cdot$ Trends over time $\cdot$ Europe $\cdot$ Time spent with friends

\section{Introduction}

Since the early 2000s, two impactful parallel transitions have taken place in the daily lives of adolescents across Europe and North America. First, substance (tobacco,

This article is part of the special issue "Adolescent transitions".

Electronic supplementary material The online version of this article (https://doi.org/10.1007/s00038-018-1182-7) contains supplementary material, which is available to authorized users.

Margaretha De Looze

M.E.deLooze@uu.nl

1 Department of Interdisciplinary Social Science, Faculty of Social and Behavioural Sciences, Utrecht University, P.O. Box 80.140, 3508 TC Utrecht, The Netherlands

2 Netherlands Institute of Mental Health and Addiction, Utrecht, The Netherlands alcohol, cannabis) use decreased substantially (De Looze et al. 2015; Hublet et al. 2015). To illustrate, data from the international Health Behaviour in School-aged Children (HBSC) study showed that the percentage of 15 year olds in Europe and North America who smoke weekly decreased from $24 \%$ in 2002 to $12 \%$ in 2014 . Weekly alcohol use decreased from $29 \%$ to $13 \%$, and lifetime cannabis use among 15 year olds decreased from $22 \%$ to 15\% (Currie et al. 2004; Inchley et al. 2016). The decline in substance use took place across virtually all countries and regions included in the study. At the same time, the so-

3 Department of Behavioral Sciences, School of Social Sciences and Humanities, Kinneret Academic College, Sea of Galilee, Israel

4 Department of Developmental and Social Psychology, University of Padova, Padova, Italy 
called digital revolution took place. The proportion of households with children that had home access to Internet increased substantially (Eurostat 2018), and rates of mobile and smart phone usage among teenagers expended enormously (Haddon et al. 2012). With the rise of social networking sites and instant messenger functions, electronic media communication (EMC) became an integral part of adolescents' daily lives (Boniel-Nissim et al. 2015a).

Some researchers suggested that the increase in EMC in the early twenty-first century can partly explain the decline in adolescent substance use (e.g. Livingston 2014; Twenge 2017). Specifically, they propose that EMC reduces the time adolescents spend face-to-face (FTF) with peers. The idea that EMC partly replaces adolescents' FTF time with peers has been referred to as the displacement hypothesis (e.g. Kraut et al. 1998; Mesch 2003; Nie 2001). As substance use typically takes place in contexts where young people socialize in person, for example, in bars and pubs at night, the displacement hypothesis poses that an increase in EMC not only leads to a decrease in FTF, but also to a decrease in substance use. While the displacement hypothesis predicts that increases over time in EMC are associated with decreases over time in FTF and substance use, the stimulation hypothesis (Valkenburg and Peter 2007a) suggests the opposite. It proposes that adolescents who are active users of EMC, spend more face-to-face time with their friends, because Internet-based communication technologies encourage communication with existing friends (e.g. Bryant et al. 2006; Kuntsche et al. 2009; Valkenburg and Peter 2007a, b, 2011). Adolescents may, for example, use EMC to communicate with peers about where and when to meet and what to do offline (Kuntsche et al. 2009; Subrahmanyam and Greenfield 2008). As a consequence, EMC may be associated with more rather than less substance use.

In sum, both the displacement and stimulation hypothesis pose that electronic media communication may affect adolescents' substance use through its influence on their time spent with friends. However, the displacement hypothesis assumes a negative effect from online communication to time spent with existing friends, whereas the stimulation hypothesis predicts a positive relationship between these variables. While early research in the field of social media use appears to confirm the displacement hypothesis (Kraut et al. 1998; Nie 2001; Nie et al. 2002; Weiser 2001), more recent research provides support for the stimulation hypothesis (Gommans et al. 2015; Kraut et al. 2002; Valkenburg and Peter 2007a, b, 2011).

A potential explanation for these inconsistent findings is that the association between EMC, FTF and substance use depends on the context (i.e. time and country) in which studies are conducted. Possibilities for communicating electronically vary considerably across countries and time.
To illustrate, in Denmark in 2010, $98 \%$ of the children aged 9-16 had Internet access at home and $75 \%$ had profiles on social networking sites. In Romania, these percentages were considerably lower (85\% and 46\%; Haddon et al. 2012). Moreover, the percentage of European youth who had access to the Internet increased significantly over time, from $67 \%$ to $96 \%$ between 2007 and 2016 (Eurostat 2018). Based on the assumption that EMC more likely functions as a stimulator of social connections in contexts where the majority of young people uses EMC, we pose that EMC relates positively to FTF in contexts where EMC is highly available to young people (i.e. in countries and time periods with high rates of EMC use). If many friends are online, young people may be more likely to use EMC to communicate with existing friends. In contrast, in contexts where a considerable part of young people is not online (yet), EMC may function more as a displacement of FTF. As such, we hypothesized that in the early 2000s and in countries where EMC is less available, EMC was associated with low FTF and consequently, low substance use. In contrast, in more recent years and in countries where large proportions of youth use EMC, we expected EMC to be positively associated with FTF and, consequently, with substance use.

\section{The present study}

This study analyses trends over time in adolescent substance use, FTF and EMC and assesses whether and how these behaviours are associated across Europe, Canada and Israel in the period 2002-2014. Using data from the international Health Behaviour in School-aged Children (HBSC) study, we aimed to answer the following research questions:

1. Are country-level trends over time in substance use associated with country-level trends over time in FTF and EMC?

2. Have associations between substance use and EMC changed across time?

3. Do associations between substance use and EMC differ across countries?

The first two research questions were analysed based on data from 2002, 2006 and 2010. Due to the introduction of new measures for EMC and FTF in 2014, the 2014 data could not be included in the trend analyses and were analysed separately. In line with recent research (Gommans et al. 2015; Kraut et al. 2002; Valkenburg and Peter 2007a, b, 2011), we overall expected to find support for the stimulation hypothesis, stating that EMC relates positively to FTF and substance use. However, we predicted that these associations varied across time and country, with 
more positive associations in more recent years and in countries with large proportions of youth using EMC.

\section{Method}

\section{Study design}

Data were obtained from the WHO collaborative HBSC study conducted in 2002-2014. HBSC is an international survey on adolescent (11-, 13-, and 15 year olds) health and the context of health, conducted every 4 years since 1983 across European and North American countries (Inchley et al. 2016). Sampling procedure and questionnaire are based on a standardized research protocol (Currie et al. 2014). Each participating country obtained approval to conduct the survey from their ethics review board or equivalent regulatory institution. Data were collected through a school-based survey, using an anonymous selfcompletion questionnaire. Under supervision of the national research teams, a translation/back-translation procedure was applied to guarantee language equivalence of the questionnaires. Representative samples on each national or regional level were selected using a clustered sampling design where the initial sampling unit was either the class or the school.

\section{Participants}

Thirty HBSC countries collected data on EMC, FTF and substance use in 2002, 2006, and 2010. Separate studies were carried out in different parts of Belgium (Flanders and Wallonia), and the UK (England, Scotland and Wales). The data for these countries were analysed at the national level. Danish data were based on mainland Denmark (data from Greenland were excluded from the analyses). In total, the analyses for tobacco and alcohol use were based on $N=$ 445,827 adolescents ( $51 \%$ girls, $M_{\text {age }}=13.5$ years old) across 26 countries. As cannabis use was assessed in 25 countries and only among 15 year olds, analyses on cannabis use were based on $N=137,398$.

In 2014, 42 HBSC countries participated in the HBSC study. Slovakia and Lithuania did not have (reliable) data on EMC; Spain and Ireland lacked data on FTF. These countries were removed from the analysis. This resulted in a sample of $N=191,727$ adolescents in 34 countries. The mean age of adolescents was 13.5 years old (ranging from 13.1 in Armenia to 13.8 in Canada). 51\% were girls (ranging from $44 \%$ in Russia to $53 \%$ in Austria and Denmark). As cannabis use was only measured among 15 year olds and two countries (Norway and Greece) did not assess cannabis use, this resulted in $N=56,159$ in 32 countries for the analyses on cannabis use.

\section{Measures}

Substance use Across all waves, substance use was assessed as follows. Weekly alcohol use. Students were asked how often they drank beer, wine, and liquor/spirits. For each type, response options were " $1=$ never", " 2 = rarely", "3 = every month", "4 = every week", "5 = every day". This variable was dichotomized by combining options 1 to 3 (indicating less than weekly alcohol use, coded as 0 ) and 4 to 5 (to reflect at least weekly drinking, coded as 1). Weekly smoking. Smoking status was defined based on the question "How often do you smoke tobacco at present?" Original answer categories (never, less than weekly, weekly but not daily, daily) were recoded into weekly smoking (1) and less than weekly smoking (0). Lifetime cannabis use. Students reported the frequency of cannabis use in their lifetime on a scale from 1 to 7 , with $1=$ never and $7=40$ times or more. Answers were recoded into $0=$ never and $1=$ at least once.

Electronic media communication (EMC) In 2002, 2006 and 2010, electronic media communication was measured by the item "How often do you talk to your friend(s) on the phone or send them text messages or have contact through the internet?" Response options were "never or rarely", "1 or 2 days a week", "3 or 4 days a week", "5 or 6 days a week", and "every day". Answers were recoded into $0=$ less than daily and $1=$ daily. In 2014, electronic media communication was measured by means of three items: How often do you a) contact your friends using texting/ SMS?; b) actively contact your friends using instant messaging (e.g. BBM, Facebook chat)?; c) contact your friends using other social media, such as Facebook (posting on wall, not chat), My Space, Twitter, apps (e.g. Instagram), games (e.g. Xbox), YouTube? Answer categories were "hardly ever or never", "less than weekly", "weekly", "daily". Answers were recoded into $0=$ less than daily and 1 = daily.

Face-to-face (FTF) contact with peers in the evening was measured by the item "How many evenings per week do you usually spend out with your friends after 8 PM?" in 2002, 2006 and 2010. The response options ranged from zero to seven evenings a week. Answers were recoded into $0=$ less than daily and $1=$ daily. In 2014 , the question was changed into "How often do you meet your friends outside school time after 8 PM?" Answer categories were hardly ever or never, less than weekly, weekly, and daily. As prevalence rates in the first categories were relatively low, 
we combined them into the category "less than once a week".

Confounders In our analyses, we controlled for age, gender and family affluence. Family affluence was measured by the ridit-based relative HBSC Family Affluence Scale (Torsheim et al. 2016).

\section{Statistical analyses}

Analyses were performed on two distinct datasets, as EMC was assessed differently in 2014, compared to the other years $(2002,2006,2010)$. Using the trend data (2002-2006-2010), we assessed descriptive statistics of trends over time in adolescent EMC, FTF, and substance use (Table 1). Correlations were calculated between two (absolute) difference scores (2002-2006 and 2002-2010) of EMC, FTF and adolescent substance use across countries. Next, hierarchical multilevel analyses (with the second-level variable being country) were performed to test the association of daily EMC with three indicators of substance use (tobacco, alcohol and cannabis; Table 2). Analyses were controlled for age (tobacco and alcohol), gender, and family affluence (tobacco). In Model 1, survey year was added to the model, followed by daily EMC (Model 2), daily FTF (Model 3), and the interaction of survey year $\times$ EMC (Model 4).

We also provide descriptive statistics of adolescent EMC, FTF, and substance use, by country, in 2014 (Table 3). Also here, hierarchical multilevel logistic regression models (with the second-level variable being country) were applied (Table 4). Analyses were controlled for age (tobacco and alcohol), gender, and family affluence (tobacco). In Model 1, daily EMC was added to the model, followed by FTF (Model 2), and the country-level mean prevalence of daily EMC (Model 3). Lastly, we added a cross-level interaction between daily EMC and the aggregated (average) EMC use per country (Model 4). Here, we divided countries into tertiles based on the percentage of youth that engaged in EMC at a daily basis. All analyses were performed using Stata SE 12.1 (College Station, Texas, USA).

\section{Results}

\section{Can the increase in EMC explain the decline in substance use between 2002 and 2010?}

Descriptive statistics of adolescent EMC, FTF and substance use in the period 2002-2010 are presented in Table 1 . Substance use decreased in virtually all countries and regions between 2002 and 2010. Some Eastern European countries (Croatia, Estonia, Latvia, Lithuania) show increasing trends for at least one substance of at least $1 \%$. FTF declined in most countries, except for Croatia, Latvia, Poland, Ukraine and Macedonia. EMC increased considerably in all countries, with increases ranging from 5.3\% (Russia) to $48.2 \%$ (Lithuania). Figure 1 in the Online appendix provides a graphical presentation of the trends per country.

When correlating the change in EMC with the change in adolescent substance use across countries across time, we did not find significant associations (see supplementary table for an overview of all correlation coefficients). While the association was significant for cannabis use between 2002 and $2010(r=0.47, p=0.02)$, we repeated the analysis leaving out Latvia and Lithuania, as they show remarkably deviant trends regarding cannabis use (i.e. a strong increase, while 18 out of 25 countries showed a decrease). This resulted in a non-significant finding ( $r=$ $0.12, p=.60$ ). Thus, even though EMC increased and substance use decreased in many countries between 2002 and 2010, the trends over time appear to be distinct and unrelated.

We also correlated country-level changes in FTF with country-level changes in substance use and EMC. Trends in EMC were not related to trends in FTF. However, positive links were found between changes in FTF and changes in substance use, especially between 2002 and 2006 (smoking: $r=.41, p=0.04$; alcohol: $r=.63, p=$ 0.0005; cannabis: $r=.42, p=0.04$ ). Between 2002 and 2010 , the trends were only associated for alcohol use ( $r=$ $.58, p=0.002$ ).

\section{EMC, FTF and substance use: different associations across time (2002-2006-2010)?}

Multilevel logistic regression models indicated that substance use overall declined between 2002 and 2006 and even more so between 2002 and 2010 (Table 2). Adolescents engaging in daily EMC and daily FTF were more likely to report substance use. Interestingly, adding EMC to the trend analysis strengthened rather than weakened the time trends in substance use. It can thus be concluded that in spite of (rather than because of) the increase in EMC, substance use decreased between 2002 and 2010. Adding FTF to the model slightly weakened the trends in adolescent substance use and substantially reduced the association between EMC and substance use. Finally, the interaction between time and EMC was not significant for smoking, but it was significant for alcohol and cannabis use. A post hoc plot showed that the positive associations between EMC and substance use were stronger in 2006 (alcohol and cannabis) and 2010 (alcohol), as compared to 2002 . 
Table 1 Adolescent face-to-face contact with peers in the evening, electronic media communication and substance use in 26 countries, 2002-2010 (\%)

\begin{tabular}{|c|c|c|c|c|c|c|c|c|c|c|c|}
\hline \multirow[t]{2}{*}{ Region } & \multirow[t]{2}{*}{ Country } & \multicolumn{2}{|c|}{$\begin{array}{l}\text { Daily face-to-face } \\
\text { contact with peers } \\
\text { in the evening }\end{array}$} & \multicolumn{2}{|c|}{$\begin{array}{l}\text { Daily electronic } \\
\text { media } \\
\text { communication }\end{array}$} & \multicolumn{2}{|c|}{ Weekly alcohol use } & \multicolumn{2}{|c|}{ Weekly tobacco use } & \multicolumn{2}{|c|}{$\begin{array}{l}\text { Lifetime cannabis } \\
\text { use }\end{array}$} \\
\hline & & 2002 & $\begin{array}{l}\text { Change } \\
02-10\end{array}$ & 2002 & $\begin{array}{l}\text { Change } \\
02-10\end{array}$ & 2002 & $\begin{array}{l}\text { Change } \\
02-10\end{array}$ & 2002 & $\begin{array}{l}\text { Change } \\
02-10\end{array}$ & 2002 & $\begin{array}{l}\text { Change } \\
02-10\end{array}$ \\
\hline \multicolumn{12}{|c|}{$\begin{array}{l}\text { Western } \\
\text { Europe }\end{array}$} \\
\hline & Austria & 5.1 & +0.8 & 23.5 & +23.1 & 9.8 & +2.0 & 12.0 & +0.1 & 13.7 & -0.1 \\
\hline & Belgium & 8.3 & -2.0 & 25.1 & +18.7 & 12.8 & -4.7 & 9.9 & -3.2 & 25.4 & -4.9 \\
\hline & France & 8.0 & -1.2 & 11.8 & +26.7 & 7.3 & -1.1 & 11.3 & -2.7 & 30.0 & -3.2 \\
\hline & Germany & 12.9 & -3.4 & 26.8 & +6.0 & 12.7 & -6.4 & 15.5 & -9.4 & 23.9 & -12.9 \\
\hline & Netherlands & 15.8 & -9.5 & 19.5 & +11.5 & 13.3 & -6.5 & 10.1 & -3.3 & 25.9 & -5.4 \\
\hline & Switzerland & 7.1 & -2.3 & 27.2 & +9.0 & 10.4 & -1.8 & 11.0 & -3.3 & 44.7 & -15.4 \\
\hline \multicolumn{12}{|c|}{$\begin{array}{l}\text { Northern } \\
\text { Europe }\end{array}$} \\
\hline & Denmark & 12.5 & -4.0 & 34.6 & +21.8 & 17.1 & -8.8 & 7.5 & -1.9 & 23.4 & -8.3 \\
\hline & Estonia & 25.2 & -6.8 & 23.8 & +17.8 & 9.6 & -3.7 & 12.3 & -3.0 & 17.2 & +4.6 \\
\hline & Latvia & 20.4 & +2.8 & 18.8 & +25.6 & 7.9 & -0.9 & 12.0 & +0.2 & 11.8 & +13.5 \\
\hline & Lithuania & 17.3 & -1.8 & 13.8 & +48.2 & 10.0 & -2.8 & 12.4 & -0.3 & 7.9 & +13.4 \\
\hline & Finland & 33.4 & -2.8 & 30.8 & +13.0 & 5.1 & -1.5 & 13.6 & -5.4 & 10.3 & -0.9 \\
\hline & Sweden & 14.2 & -1.8 & 26.9 & +26.4 & 6.8 & -4.4 & 6.6 & -1.1 & 6.8 & - \\
\hline & UK & 33.7 & -10.2 & 33.7 & +15.9 & 22.3 & -12.2 & 10.8 & -4.8 & 37.6 & -17.4 \\
\hline \multicolumn{12}{|c|}{$\begin{array}{l}\text { Eastern } \\
\text { Europe }\end{array}$} \\
\hline & Czech Rep. & 16.4 & -2.6 & 23.5 & +16.8 & 19.2 & +0.8 & 14.2 & -1.8 & 30.5 & +0.0 \\
\hline & Estonia & 25.2 & -6.8 & 23.8 & +17.8 & 9.6 & -3.7 & 12.3 & -3.0 & 17.2 & +4.6 \\
\hline & Poland & 13.3 & +1.6 & 19.5 & +28.3 & 7.4 & -1.4 & 11.2 & -3.7 & 18.1 & +0.7 \\
\hline & Russia & 35.6 & -1.8 & 46.4 & +5.3 & 14.3 & -8.3 & 12.3 & -2.7 & 13.6 & -5.2 \\
\hline & Ukraine & 28.0 & +2.1 & 24.9 & +19.4 & 18.7 & -2.6 & 16.7 & -7.6 & 23.4 & -12.4 \\
\hline & Macedonia & 13.9 & +4.3 & 36.2 & +9.7 & 6.8 & +0.6 & 5.7 & -0.2 & 3.1 & -0.3 \\
\hline \multicolumn{12}{|c|}{$\begin{array}{r}\text { Southern } \\
\text { Europe }\end{array}$} \\
\hline & Croatia & 13.2 & +2.9 & 42.7 & +5.5 & 13.3 & +3.4 & 9.7 & +2.7 & 16.2 & -2.8 \\
\hline & Greece & 9.0 & +0.2 & 38.2 & +7.2 & 16.1 & -2.0 & 6.2 & +0.2 & 5.2 & +1.8 \\
\hline & Italy & 10.7 & -1.7 & 34.7 & +14.2 & 23.0 & -10.9 & 10.0 & -0.8 & 21.4 & -1.8 \\
\hline & Portugal & 5.0 & -2.4 & 25.9 & +29.2 & 8.0 & -4.2 & 11.3 & -6.1 & 19.8 & -8.5 \\
\hline & Slovenia & 12.1 & -2.0 & 33.6 & +8.2 & 10.8 & +0.4 & 10.3 & -2.7 & 28.4 & -5.2 \\
\hline \multicolumn{12}{|c|}{ Non-European } \\
\hline & Canada & 24.1 & -3.4 & 40.5 & +7.0 & 10.6 & -4.9 & 6.8 & -1.3 & 44.3 & -10.4 \\
\hline & Israel & 20.2 & -8.4 & 42.3 & +11.2 & 15.3 & -1.9 & 7.9 & -1.4 & 5.9 & -0.5 \\
\hline
\end{tabular}

-, No data available

\section{EMC, FTF and substance use in 2014: different associations across countries?}

Descriptive statistics of EMC, FTF, and substance use in 2014 are presented in Table 3. EMC and FTF differed considerably across countries, with EMC ranging from $27.6 \%$ in the Republic of Moldova to $88.1 \%$ in Switzerland and FTF ranging from 2.2\% (Portugal) to $33.7 \%$ (Bulgaria). Weekly alcohol use ranged from $1.9 \%$ (Iceland) to $16.3 \%$ (Bulgaria). Weekly tobacco use ranged from $1.5 \%$ (Iceland) to $11.5 \%$ (Bulgaria) and lifetime cannabis use among 15 year olds ranged from $1.5 \%$ (Armenia) to $28.2 \%$ (Belgium). 


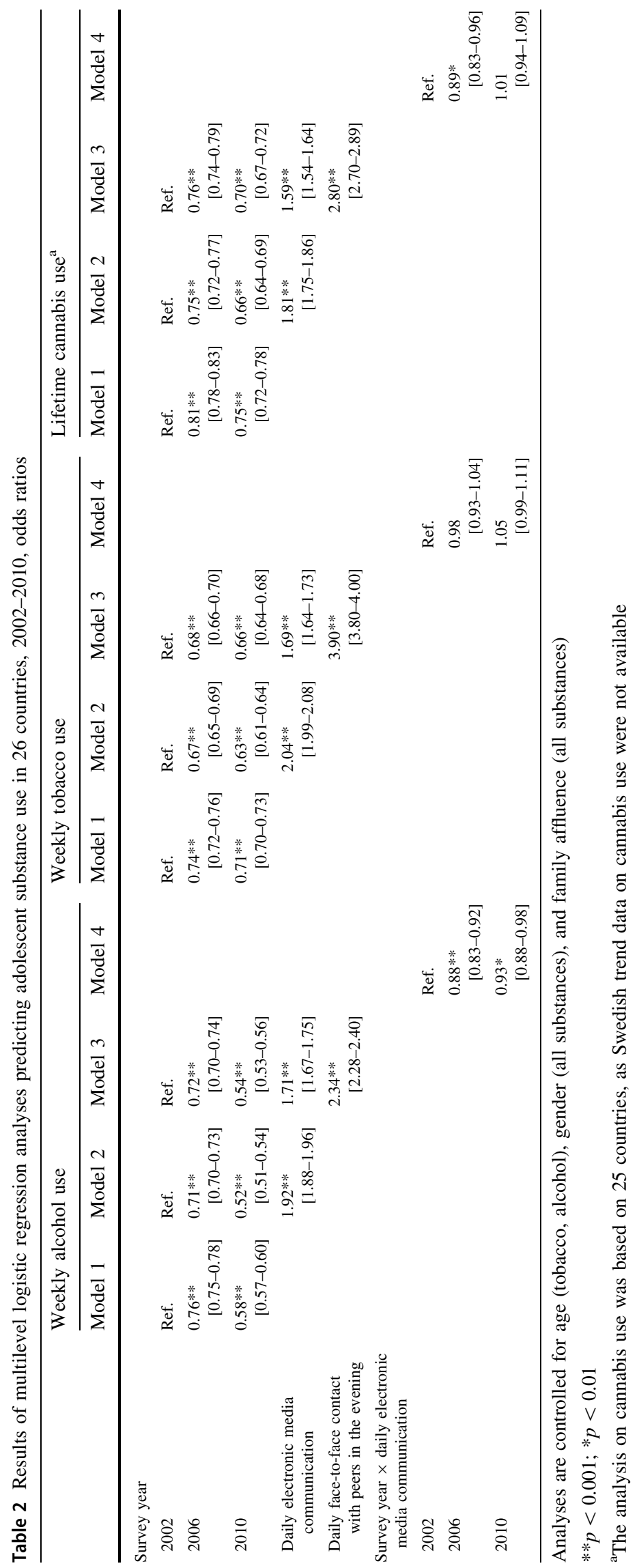


Table 3 Adolescent face-to-face contact with peers in the evening, electronic media communication and substance use in 34 countries in 2014 , $\%$

\begin{tabular}{|c|c|c|c|c|c|c|}
\hline Region & Country & $\begin{array}{l}\text { Daily face-to-face contact with peers } \\
\text { in the evening }\end{array}$ & $\begin{array}{l}\text { Daily electronic media } \\
\text { communication }\end{array}$ & $\begin{array}{l}\text { Weekly } \\
\text { alcohol use }\end{array}$ & $\begin{array}{l}\text { Weekly } \\
\text { tobacco use }\end{array}$ & $\begin{array}{l}\text { Lifetime } \\
\text { cannabis use }\end{array}$ \\
\hline \multicolumn{7}{|c|}{$\begin{array}{l}\text { Western } \\
\text { Europe }\end{array}$} \\
\hline & Austria & 5.2 & 83.5 & 8.7 & 6.6 & 9.5 \\
\hline & Belgium & 3.7 & 64.4 & 6.6 & 5.0 & 17.7 \\
\hline & France & 5.0 & 76.6 & 6.6 & 7.8 & 28.2 \\
\hline & Germany & 4.4 & 64.6 & 6.5 & 6.2 & 16.7 \\
\hline & Luxembourg & 12.4 & 64.8 & 7.1 & 8.0 & 18.0 \\
\hline & Netherlands & 5.3 & 63.5 & 5.9 & 4.4 & 15.9 \\
\hline & Switzerland & 2.9 & 88.1 & 4.4 & 4.5 & 23.7 \\
\hline \multicolumn{7}{|c|}{$\begin{array}{r}\text { Northern } \\
\text { Europe }\end{array}$} \\
\hline & Denmark & 5.2 & 63.4 & 6.2 & 4.1 & 15.7 \\
\hline & Estonia & 4.8 & 58.0 & 4.5 & 5.4 & 24.3 \\
\hline & Latvia & 6.2 & 50.9 & 3.6 & 5.8 & 20.5 \\
\hline & Finland & 7.9 & 82.7 & 4.8 & 5.8 & 9.6 \\
\hline & Norway & 11.9 & 53.6 & 1.5 & 1.8 & - \\
\hline & Sweden & 5.3 & 63.9 & 2.7 & 2.9 & 5.8 \\
\hline & UK & 8.7 & 68.5 & 6.0 & 3.6 & 17.9 \\
\hline \multicolumn{7}{|c|}{$\begin{array}{l}\text { Eastern } \\
\text { Europe }\end{array}$} \\
\hline & Albania & 15.8 & 51.3 & 11.6 & 2.8 & 5.5 \\
\hline & Armenia & 14.5 & 50.7 & 10.2 & 1.8 & 1.5 \\
\hline & Bulgaria & 33.7 & 72.1 & 16.3 & 11.5 & 22.7 \\
\hline & Czech Rep. & 3.6 & 38.7 & 9.6 & 6.3 & 23.1 \\
\hline & Hungary & 13.1 & 57.0 & 11.6 & 7.9 & 12.9 \\
\hline & Macedonia & 22.0 & 70.8 & 7.2 & 4.1 & 3.2 \\
\hline & $\begin{array}{l}\text { Rep. of } \\
\text { Moldova }\end{array}$ & 8.5 & 27.6 & 12.4 & 3.6 & 5.7 \\
\hline & Poland & 6.9 & 54.5 & 6.4 & 8.0 & 23.6 \\
\hline & Romania & 17.5 & 41.0 & 13.7 & 9.6 & 7.5 \\
\hline & Russia & 14.2 & 64.2 & 6.0 & 8.6 & 8.6 \\
\hline & Ukraine & 11.3 & 48.3 & 13.7 & 5.7 & 7.2 \\
\hline \multicolumn{7}{|c|}{$\begin{array}{r}\text { Southern } \\
\text { Europe }\end{array}$} \\
\hline & Croatia & 12.5 & 64.9 & 14.1 & 9.5 & 15.2 \\
\hline & Greece & 14.0 & 54.2 & 11.6 & 5.7 & - \\
\hline & Italy & 7.6 & 80.4 & 13.4 & 8.6 & 22.0 \\
\hline & Malta & 4.8 & 53.1 & 11.1 & 4.4 & 13.3 \\
\hline & Portugal & 2.2 & 33.6 & 4.4 & 4.2 & 11.7 \\
\hline & Slovenia & 2.9 & 47.4 & 8.6 & 5.1 & 21.1 \\
\hline \multicolumn{7}{|c|}{$\begin{array}{l}\text { Non- } \\
\text { European }\end{array}$} \\
\hline & Canada & 11.2 & 59.4 & 5.7 & 3.3 & 25.5 \\
\hline & Israel & 9.2 & 63.9 & 13.2 & 6.7 & 6.5 \\
\hline
\end{tabular}

- , No data available 


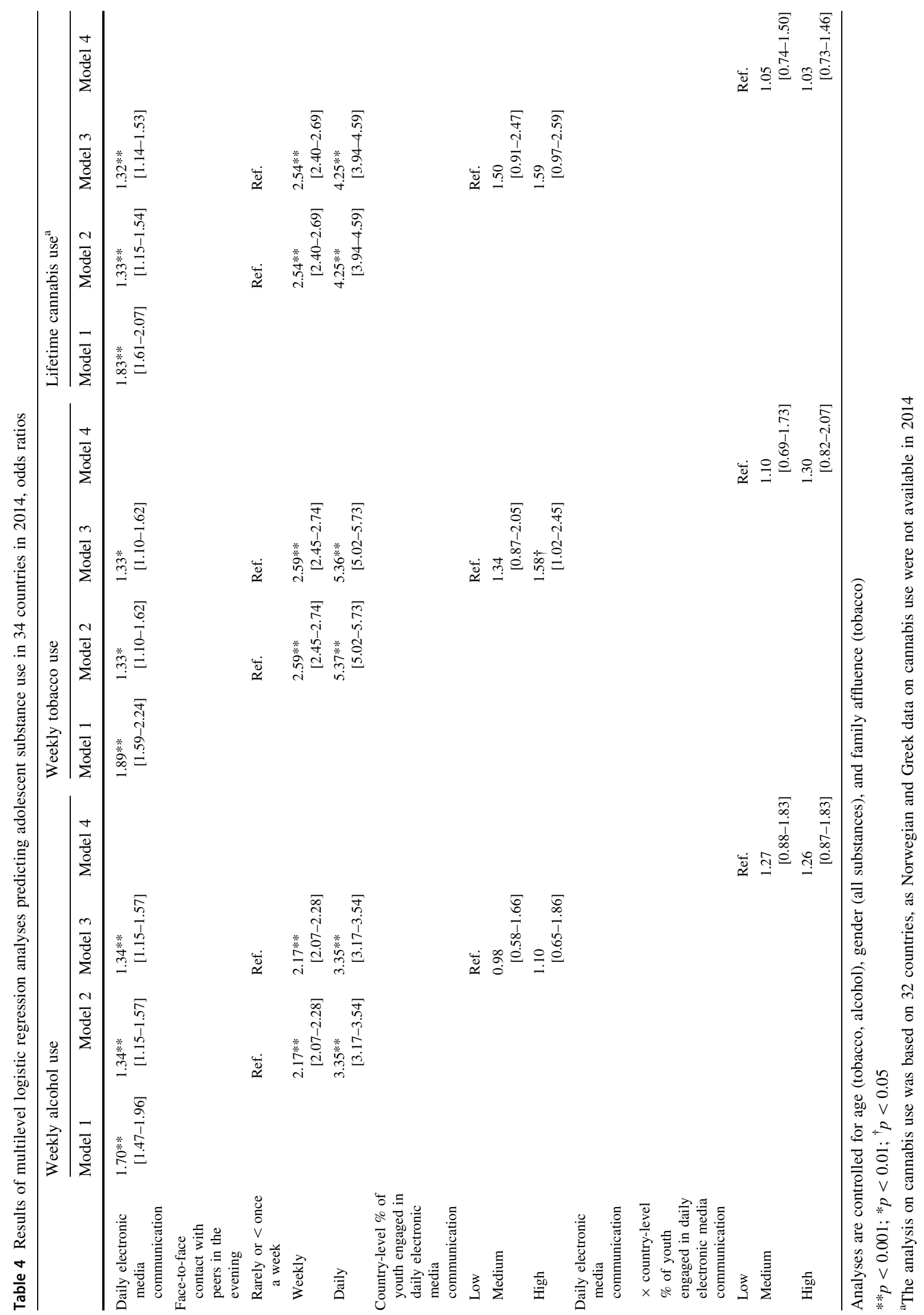


Hierarchical multilevel logistic regression models were applied to test the association of EMC with substance use across countries while controlling for age, gender, and family affluence (Table 4). Overall, EMC was positively associated with the three types of substance use (Model 1, Table 4). FTF was also positively associated with all three substances (Model 2, Table 4). Adding FTF to the model reduced the strength of the associations between EMC and substance use, but all associations remained significant. Thus, FTF partly explained the positive association between EMC and substance use.

The next step was to examine if the association between EMC and substance use differed across countries, and if so, if this cross-national variation could be explained by the popularity of EMC among youth in a given country. We first examined whether there was sufficient variability between countries to justify a random slope for EMC. The difference in log likelihood between a model with a random and a fixed slope was significant $(p s<0.01)$, indicating that associations between EMC and substance use differed across countries. To test whether the cross-national variation in these associations could be explained by the country-level popularity of EMC among youth, we added the country-level mean prevalence of daily EMC and its interaction with individual EMC as predictors to the model (Model 3 and 4, respectively). The interactions between individual daily EMC and the average country-level EMC were not significant, indicating that the cross-national variability in the association between EMC and substance use cannot be explained by the country-level popularity of EMC among adolescents.

To gain more insight into the cross-national differences in the association between EMC and substance use, we examined these associations in each country separately (not in a table). In the majority of countries, associations were positive, but in a few countries, there was a negative association between EMC and alcohol use (Russian Federation, Israel, Iceland) and tobacco use (Israel).

\section{Supplementary analyses}

As age and gender relate strongly to adolescent substance use and EMC, we examined whether the associations found in this study differed across age groups and between boys and girls. Interaction terms (gender $\times$ EMC; age $\times$ EMC) were added to Model 1 of Table 4. The association of EMC with smoking was stronger for girls than for boys $(\mathrm{OR}=$ $1.26, p<0.001)$, but no gender differences were found in the associations with alcohol and cannabis. For both smoking and alcohol, associations with EMC were stronger for older adolescents (ORs up to 1.76, $p$ s $<0.001$ ). For cannabis, no interaction with age was performed as it was only measured among 15 year olds.

\section{Discussion}

This study provides no support for the hypothesis that recent declines in adolescent substance use, reported in the majority of western countries, can be explained by increasing EMC among adolescents during the same period. First, on a national level, declines in substance use over time were not associated with increases in EMC, while they were associated with declines in FTF. Second, across countries and time, adolescents who reported daily EMC spent more time with friends in the evenings and were more likely to use substances, than adolescents who did not report daily EMC. Although positive associations between EMC and substance use were found in the vast majority of countries, the associations varied between countries. This cross-national variation could not be explained by the country-level use of EMC among youth. Finally, for alcohol and cannabis use, the positive association with EMC varied in strength across time, with stronger associations in more recent survey years.

Overall, our findings provide support for the stimulation rather than the displacement hypothesis, suggesting that EMC overall functions as a social connector for adolescents. It seems to intensify already existing friendships, rather than replace face-to-face peer interactions. As adolescents often engage in substance use in the presence of peers (Branstetter et al. 2011; Chassin et al. 2009), adolescents who tend to use EMC are may be more likely to report substance use.

In line with our hypotheses, the positive links between EMC and substance use were stronger in more recent years, although this was primarily the case for alcohol (2006 and 2010) and cannabis use (2006), as compared to 2002. The idea behind this hypothesis was that increased use of EMC enhances the function of EMC as a social connector which, in turn, affects the association with substance use. There are several reasons why these results were particularly revealed for alcohol use. First, we have reason to believe that alcohol use is typically consumed for social reasons by young people (Kuntsche et al. 2005), making associations between EMC and alcohol use more likely in contexts in which EMC serves more as a social connector. Second, the increased popularity of EMC over time has led to an increase in online advertisements, especially for alcohol (Anderson et al. 2009; Nicholls 2012; Pennay et al. 2015), leading to an increased association of EMC to alcohol use. Third, many adolescents use EMC to display engagement in substance use, and the most frequently displayed substance is alcohol (e.g. texting about or posting pictures of partying and drinking; Loss et al. 2013). The more prevalent EMC became, the more adolescents may have been 
exposed to such displays, which may have strengthened the association between EMC and alcohol use.

In contrast to our hypotheses, the link between EMC and substance use did not vary with the prevalence of EMC in a particular country. Potentially, social norms regarding substance use in different countries have a larger effect on this link than the prevalence of EMC. For example, the association between EMC and substance use may depend on the extent to which substance use is part of popular youth culture. In countries with relatively low prevalence rates of substance use (such as Iceland), the association with EMC may be different as substance use is not integrated in popular youth culture, while EMC is. Moreover, the extent to which parents supervise the EMC of their children, or the extent to which it is culturally accepted that adolescents spend (unsupervised) time together at night with friends, may differ across countries. Such factors are also likely to affect links between EMC, FTF, and substance use.

Although we predominantly found positive associations between EMC and substance use across countries and time, our study does not rule out that EMC is related to less FTF and less substance use for specific groups of adolescents. For example, socially anxious and lonely adolescents seem to prefer EMC over FTF communication (Pierce 2009) and more strongly value the opportunity to communicate anonymously online (Valkenburg and Peter 2007b, 2011). This suggests that for socially anxious and lonely adolescents, EMC does not necessarily lead to new friendships, more FTF or more substance use. Future research is needed to gain more insight into these individual differences in the potential effects of EMC on adolescent substance use.

Our findings suggest that, rather than an increase in EMC, a decrease in FTF may have played a role in the declining trends in adolescent substance use between 2002 and 2010. Among others, the decrease in FTF in the evening might be the consequence of increasingly strict parental rule-setting and monitoring, which has been reported in some European countries (e.g. De Looze et al. 2017) and in the USA (Twenge 2017). If parents are less likely to allow their children to go out at night, they restrict their children's exposure to contexts in which substances are typically used. Potentially, the rise of the Internet technology in a broader sense has enabled parents to monitor their children more easily, for instance because it has become more common for children to have their own cell phone. The increase in substance use prevention programs across countries (Kuntsche and Ravens-Sieberer 2015) and stricter rules regarding the use and purchase of substance use across Europe (e.g. ban of smoking in public areas, ban of selling tobacco and alcohol to minors) may also have encouraged parents to become stricter regarding their child's exposure to contexts that could lead to substance use. An alternative explanation for the decline in FTF in the evenings may be that youth are becoming more involved in structured and constructive activities during the day. While evidence from the USA does not seem to support this hypothesis (Twenge 2017), European trend data on leisure time use are scarce.

\section{Strengths and limitations}

A major strength of this study lies in the use of large and nationally representative samples of adolescents in a sizeable number of countries which are heterogeneous in terms of, for example, their sociocultural and policy backgrounds. Moreover, our study compromises a time span of 12 years, which provided us with the unique opportunity to correlate national-level changes in EMC, FTF and substance use over time. The inclusion of data from different countries and different moments in time increases the external validity of our study. However, this study should be interpreted with knowledge of its limitations. First, due to the repeated cross-sectional design, no causal inferences can be made. Adolescents who are at risk of substance use may also be at risk of higher levels of EMC (Osaki et al. 2012), or adolescents who use substances use may be more drawn to EMC because their substance use enhances their social connectedness and standing among peers (KilleyaJones et al. 2007). Second, although we included an important set of social and individual factors in our models and showed that they contributed to substance use independently, a more elaborate model of substance use should also include biological, genetic and personality factors, as well as their interactions. Such a model provides opportunities for examining whether EMC use is differently associated with substance use for specific groups of adolescents (e.g. excessive users; active versus passive users; socially anxious adolescents; (e.g. Blinka et al. 2015; Boniel-Nissim et al. 2015b). Third, while adolescents are fairly accurate in their self-reported substance use (e.g. Harrison et al. 2007), the self-reported nature of all measures in this study may have led to biased estimates.

\section{Conclusion}

Not the increase in EMC, but rather the decrease in FTF may have played a role in the decline in adolescent substance use in the early twenty-first century. This study suggests that adolescents who engage in daily EMC interact with friends relatively often, online as well as offline, and are more likely to engage in substance use. We call for future studies to replicate our findings and examine links between EMC, FTF and substance use in a longitudinal design. 


\section{Compliance with ethical standards}

Conflict of interest The authors declare that they have no conflict of interest.

Ethical approval Each country that participated in the HBSC study obtained approval to conduct the survey from their ethics review board or equivalent regulatory body.

Informed consent Participation was voluntary, and informed consent (active or passive) was sought from school administrators, parents and children as per national human subject requirements.

Open Access This article is distributed under the terms of the Creative Commons Attribution 4.0 International License (http://creative commons.org/licenses/by/4.0/), which permits unrestricted use, distribution, and reproduction in any medium, provided you give appropriate credit to the original author(s) and the source, provide a link to the Creative Commons license, and indicate if changes were made.

\section{References}

Anderson P, de Bruijn A, Angus K, Gordon R, Hastings G (2009) Impact of alcohol advertising and media exposure on adolescent alcohol use: a systematic review of longitudinal studies. Alcohol Alcohol 44(3):229-243

Blinka L, Škařupová K, Ševčíková A, Wölfling K, Müller KW, Dreier M (2015) Excessive internet use in European adolescents: What determines differences in severity? Int $\mathbf{J}$ Public Health 60:249-256

Boniel-Nissim M, Lenzi M, Zsizos E, Gaspar de Matos M, Gommans R, Harel-Fisch Y, Djalovski A, Van der Sluijs W (2015a) International trends in electronic media communication (EMC) among 11- to 15-year-olds (2002-2010): Does EMC affect ease of communication with friends from the opposite sex? Eur J Public Health 25:41-45

Boniel-Nissim M, Tabak I, Mazur J, Borraccino A, Brooks F, Gommans R, van der Sluijs W, Zsiros E, Craig W, Harel-Fisch Y, Finne E (2015b) Supportive communication with parents moderates the negative effects of electronic media use on life satisfaction during adolescence. Int J Public Health 60:189-198

Branstetter SA, Low S, Furman W (2011) The influence of parents and friends on adolescent substance use: multidimensional approach. J Subst Use 16:150-160

Bryant JA, Sanders-Jackson A, Smallwood AMK (2006) IMing, text messaging, and adolescent social networks. J Comput Mediat Commun 11:577-592

Chassin L, Hussong A, Barrera M Jr, Molina BSG, Trim R, Ritter J (2009) Adolescent substance use. In: Lerner R, Steinberg L (eds) Handbook of adolescent psychology, 3rd edn. Wiley, Hoboken, pp 723-763

Currie C, Roberts C, Morgan A, Smith R, Settertobulte W, Samdal O, Barnekow V (eds.) (2004) Young people's health in context: International report from the HBSC 2001/02 survey, (Health Policy for Children and Adolescents, No.4). WHO Regional Office for Europe, Copenhagen.

Currie C, Inchely J, Molcho M et al (eds) Health Behavior in Schoolaged Children (HBSC) study protocol: background, methodology and mandatory items for the 2013/14 survey St Andrews, Scotland: Child and Adolescent Health Research Unit, University of St Andrews, 2014 (http://www.hbscorg/news/index aspx?ni=2418. Accessed 1 April 2018
De Looze M, Raaijmakers Q, ter Bogt T, Bendsten P, Farhat T, Ferreira M, Godeau E, Kuntsche E, Molcho M, Pförtner TK, Simons-Morton B, Vollebergh W, Pickett W (2015) Decreases in weekly alcohol use in Europe and North America: evidence from 28 countries from 2002 to 2010. Eur J Public Health 25(2Suppl):69-72

De Looze M, van Dorsselaer S, Monshouwer K, Vollebergh W (2017) Trends in adolescent alcohol use in the Netherlands, 1992-2015. Differences across sociodemographic groups and links with strict parental rule-setting. Int J Drug Policy 50:90-101

Eurostat (2018) Internet access and use statistics - households and individuals. Available at http://ec.europa.eu/eurostat/statisticsexplained/index.php/Internet_access_and_use_statistics_-_house holds_and_individuals

Gommans R, Stevens GWJM, Finne E et al (2015) Frequent electronic media communication with friends is associated with higher adolescent substance use. Int J Public Health 60:167-177

Haddon L, Livingstone S, Online Kids, EU Kids Online network (2012) EU Kids Online: national perspectives, EU Kids Online. The London School of Economics and Political Science, London

Harrison LD, Martin SS, Enev T, Harrington D (2007) Comparing drug testing and self-report of drug use among youths and young adults in the general population (DHHS Publication No. SMA 07-4249, Methodology Series M-7). Rockville, MD: Substance Abuse and Mental Health Services Administration, Office of Applied Studies

Hublet A, de Looze M, Fotiou A, Donnelly P, Vilhjalmsson R, Baska T, Aasvee K, Franelic IP, Nic Gabhainn S, ter Bogt T (2015) Trends in the co-occurrence of tobacco and cannabis use in 15-year-olds from 2002 to 2010 in 28 countries of Europe and North America. Eur J Public Health 25(Suppl2):73-75

Inchley J, Currie D, Young T et al (eds) (2016) Growing up unequal: gender and socioeconomic differences in young people's health and well-being Health Behaviour in School-aged Children (HBSC) study: international report from the 2013/2014 survey. WHO Regional Office for Europe, Copenhagen

Killeya-Jones LA, Nakajima R, Costanzo PR (2007) Peer standing and substance use in early-adolescent grade-level networks: a short-term longitudinal study. Prev Sci 8:11-23

Kraut R, Patterson M, Lundmark V et al (1998) Internet paradox: a social technology that reduces social involvement and psychological well-being? Am Psychol 53:1017-1031

Kraut R, Kiesler S, Boneva B et al (2002) Internet paradox revisited. J Soc Issues 58:49-74

Kuntsche E, Ravens-Sieberer U (2015) Monitoring adolescent health behaviours and social determinants cross-nationally over more than a decade: introducing the Health Behaviour in School-aged Children (HBSC) study supplement on trends. Eur J Public Health 25(Suppl 2):1-3

Kuntsche E, Knibbe R, Gmel G, Engels R (2005) Why do young people drink? A review of drinking motives. Clin Psychol Rev 27(7):841-861

Kuntsche E, Simons-Morton B, ter Bogt T et al (2009) Electronic media communication with friends from 2006 to 2010 and links to face-to-face contacts in adolescence: an HBSC study in 31 European and North American countries and regions. Int J Public Health 54(Suppl 2):243-250

Livingston M (2014) Trends in non-drinking among Australian adolescents. Addiction 109(6):922-929

Loss J, Lindacher V, Curbach J (2013) Do social networking sites enhance the attractiveness of risky health behaviour? Impression management in adolescents' communication on Facebook and its ethical implications. Public Health Issues 7(1):5-16

Mesch GS (2003) The family and the internet: the Israeli case. Soc Sci Q 84:1038-1050 
Nicholls J (2012) Everyday, everywhere: alcohol marketing and social media-current trends. Alcohol Alcohol 47:486-493

Nie NH (2001) Sociability, interpersonal relationships and the internet: reconciling conflicting findings. Am Behav Sci 45:420-435

Nie NH, Hillygus DS, Erbring L (2002) Internet use, interpersonal relations, and sociability: a time diary study. In: Wellman B, Haythornthwaite $\mathrm{C}$ (eds) The internet in everyday life. Blackwell, Oxford, pp 215-243

Osaki Y, Ohida T, Kanda H, Kaneita Y, Kishimoto T (2012) Mobile phone use does not discourage adolescent smoking in Japan. Asian Pac J Cancer Prev 13:1011-1014

Pennay A, Livingston M, MacLean S (2015) Young people are drinking less: it is time to find out why. Drug Alcohol Rev 34(2):115-118

Pierce T (2009) Social anxiety and technology: face-to-face communication versus technological communication among teens. Comput Hum Behav 25:1367-1372

Subrahmanyam K, Greenfield P (2008) Online communication and adolescent relationships. Future Child 18:119-146

Torsheim T, Cavallo F, Levin KA et al (2016) Psychometric validation of the revised family affluence scale: a latent variable approach. Child Indic Res 9:771-784
Twenge JM (2017) Why today's super-connected kids are growing up less rebellious, more tolerant, less happy-and completely unprepared for adulthood and what that means for the rest of us. Atria Books, New York

Valkenburg PM, Peter J (2007a) Online communication and adolescent well-being: testing the stimulation versus the displacement hypothesis. J Comput Mediat Commun 12:1169-1182

Valkenburg PM, Peter J (2007b) Preadolescents' and adolescents' online communication and their closeness to friends. Dev Psychol 43:267-277

Valkenburg PM, Peter J (2011) Online communication among adolescents: an integrated model of its attraction, opportunities, and risks. J Adol Health 48:121-127

Weiser EB (2001) The functions of Internet use and their psychological consequences. Cyberpsychol Behav 4(6):723-744

Publisher's Note Springer Nature remains neutral with regard to jurisdictional claims in published maps and institutional affiliations. 